\title{
On the (De)homogenization of Sagbi Bases
}

\author{
Junaid Alam Khan
}

\begin{abstract}
In this paper we study the relation between nonhomogeneous and homogeneous Sagbi bases. As a consequence, we present a general principle of computing Sagbi bases of a subalgebra and its homogenized subalgebra, which is based on passing over to homogenized generators.
\end{abstract}

\section{Introduction}

Our interest in the subject of this paper is inspired by $[3,6,7]$, where they address the problem of behavior of Gröbner bases $([1,2])$ under homogenization and dehomogenization.

Let $R=K\left[x_{1}, \ldots, x_{n}\right]$, denote the polynomial ring over the field $K$ and $R[t]$ be the polynomial ring in the variable " $t$ " over $R$. The concept of Gröbner bases for ideals of a polynomial ring over a field $K$ can be adapted in a natural way to $K$-subalgebras of a polynomial ring. In [11] Sagbi (Subalgebra Analog to Gröbner Basis for Ideals) basis for the $K$-subalgebra of $R$ are defined, this concept was independently developed in [5]. It is shown in $[5,11]$ that Sagbi bases play the same computational role for subalgebras as Gröbner bases play for ideals. Miller extended Sagbi basis theory to polynomial rings over a commutative Noetherian domain in [8]. Since many of the basic concepts of Gröbner basis apply to the subalgebra case, it is natural to ask about the behavior of Sagbi basis under homogenization and dehomogenization. In this paper, we employ the homogenization and dehomogenization technique to study in detail the relation of Sagbi bases in $R$ and homogenous Sagbi bases

Key Words: Sagbi basis, Homogenous polynomial.

2010 Mathematics Subject Classification: Primary 13P10.

Received: February 2012.

Revised: March 2012.

Accepted: July 2012. 
in the polynomial ring $R[t]$. Other notable works in related themes of study include [9].

The work presented herein is contained in two main sections. In Section 2, we give some basic notations and definitions related to Sagbi bases. Then in Section 3, we show that the application of homogenizing and dehomogenizing properties of polynomials to subalgebras provide good properties for homogenizing subalgebras (Theorem 3.3). After that, we examine the behavior of Sagbi bases of subalgebras under homogenization and dehomogenization and show that Sagbi bases homogenize and dehomogenize in a very natural way (Theorem 3.5 and 3.6). Finally, we discuss the behavior of s-reduced Sagbi bases under homogenization and dehomogenization.

\section{Notation and definition}

In this section, we will review some basic terminology and results of Sagbi basis theory that will be used in the subsequent section. The reader who is familiar with the theory is still encouraged to skim through this section in order to get familiar with the notational convention.

By a monomial in $R$, we mean an element of the form $x_{1}^{\alpha_{1}} \ldots x_{n}^{\alpha_{n}}$ with $\alpha_{1}, \ldots, \alpha_{n} \in \mathbb{N}=\{0,1,2, \ldots\}$, we denote the set of all monomials by $\operatorname{Mon}(R)$. Note that $1=x_{1}^{0} \ldots x_{n}^{0} \in \operatorname{Mon}(R)$.

Let $R_{j}$ be the $K$-vector space spanned by all homogeneous polynomials of degree $j$. The vector space $R_{j}$ is finite-dimensional, and the monomials $u$ with $\operatorname{deg}(u)=j$ form a $K$-basis of this vector space. Moreover, $R=\bigoplus_{j} R_{j}$. Therefore, each polynomial $f \in R$ can be uniquely written as $f=\sum_{j} f_{j}$ with $f_{j} \in R_{j}$. The summands $f_{j}$ are called the homogeneous components of $f$. If $i=\operatorname{deg}(f)$, then $f_{i}$ is called the leading homogenous polynomial of $f$, and is denoted by $L H(f)$.

If $G$ is a subset of $R$ (not necessarily finite), then the subalgebra of $R$ generated by $G$ is usually denoted by $K[G]$. This is natural notation since the elements of $K[G]$ are precisely the polynomials in the set of formal variables $G$, viewed as elements of $K[G]$.

In this paper, we always assume that monomial orderings are global monomial orderings, i.e., well orderings. Let $>$ be a monomial ordering on $\operatorname{Mon}(R)$ (see for e.g. [3]). We can associate to every non-zero polynomial $f \in R$ its leading monomial, denoted by $L M_{>}(f)(\in \operatorname{Mon}(R))$. We call the coefficient of $L M_{>}(f)$ the leading coefficient of $f$ denoted by $L C_{>}(f), L C_{>}(f) L M_{>}(f)$ is called the leading term and denoted by $L T_{>}(f)$. We also define, for a subset $G \subset R, L M_{>}(G)=\left\{L M_{>}(f) \mid f \in G\right\}$. For $f \in R$, support $(f)$ is the set of all monomials contained in $f$. 
Note that $R[t]=\bigoplus_{p} R_{p}[t]$ is an $\mathbb{N}$-graded algebra with the degree- $p$ homogenous part

$$
R[t]_{p}=\left\{\sum_{i+j=p} F_{i} t^{j} \mid F_{i} \in R_{i}, j \geq 0\right\}, \quad p \in \mathbb{N}
$$

A monomial ordering $>$ is said to be a degree ordering if $\operatorname{deg}(u)>\operatorname{deg}(v)$ for $u, v \in \operatorname{Mon}(R)$, implies $u>v$. For a degree ordering $>$ we define a natural extension $>_{t}$ to $R[t]$ as follows:

$$
x^{a} t^{c}>_{t} x^{b} t^{d} \text { if and only if } x^{a}>x^{b} \text {, or } x^{a}=x^{b} \text { and } c>d
$$

From now on we assume that all monomial orderings considered are degree orderings. Here we gather some definitions concerning Sagbi bases that we will need. For a more complete exposition we refer to [11].

Definition 2.1. Let $G$ be a subset of $R$ or $R[t]$ and $P=K\left[y_{1}, \ldots, y_{m}\right]$. For $m=y_{1}^{\alpha_{1}} \ldots y_{r}^{\alpha_{r}} \in \operatorname{Mon}(P)$, a $G$-monomial is a finite power product of the form $m(G)=g_{1}^{\alpha_{1}} \ldots g_{r}^{\alpha_{r}}$ where $g_{i} \in G$ for $i=1, \ldots, r$, and $\alpha_{1}, \ldots, \alpha_{r} \in \mathbb{N}$. We denote the set of all $G$-monomials by $\operatorname{Mon}(G)$.

Definition 2.2. A (possibly infinite) subset $S$ of $K[G]$ is called Sagbi basis of $K[G]$ with respect to $>$ if

$$
K\left[L M_{>}(K[G])\right]=K\left[L M_{>}(S)\right]
$$

In other words for any $f \in K[G]$ there exist an $m(S) \in M o n(S)$ such that $L M_{>}(f)=L M_{>}(m(S))$.

Definition 2.3. Let $G$ be a Sagbi basis of the subalgebra $A$ with respect to $>$. We say that $G$ is the s-reduced Sagbi basis of $A$ if the following conditions are satisfied:

(1) For all $g \in G, L C_{>}(g)=1$ and $L M_{>}(g) \notin K\left[L M_{>}(G \backslash\{g\})\right]$.

(2) For all $g \in G$, we have $\operatorname{support}\left(g-L M_{>}(g)\right) \cap K\left[L M_{>}(G)\right]=\varnothing$.

\section{3 (De)homogenized Sagbi basis}

Let $f \in R$ we denote by $f^{*}$ the homogenization of $f$ with respect to $t$ in $R[t]$. For $F \in R[t]$, let $F_{*}=F_{t=1}$ be the dehomogenization of $F$ with respect to $t$. For $G \subset R, G^{*}=\left\{f^{*} \mid f \in R\right\}$.

Considering the onto ring homomorphism $\phi: R[t] \rightarrow R$ defined by $\phi(t)=1$, then for each $f \in R[t]$, there exist $f^{*} \in R[t]$ satisfying $\phi\left(f^{*}\right)=f$. On the other hand for $F \in R[t]$, we write $\phi(F)=F_{*}$. 
Definition 3.1. Let $A$ be a subalgebra in $R$ and $B$ be a subalgebra in $R[t]$. (a) The subalgebra $A^{*}=K\left[\left\{f^{*} \mid f \in A\right\}\right]$ in $R$ is called the homogenization of $A$ with respect to the variable $t$.

(b) The set $B_{*}=\left\{f_{*} \mid f \in A\right\}$ in $R$ is called the dehomogenization. The set $B_{*}$ is a subalgebra of $R$.

Let $G$ be a nonempty subset of $R$ and $A=K[G]$ the subalgebra generated by $G$. Next example shows that, in general $K\left[G^{*}\right] \subset A^{*}$.

Example 3.2. Let $G=\left\{f_{1}, f_{2}\right\} \subset K[x, y, z]$ where $f_{1}=x^{3}+y$ and $f_{2}=x^{3}-z$. Let $A=K[G]=K\left[f_{1}, f_{2}\right]$. Then $K\left[G^{*}\right]=K\left[f_{1}^{*}, f_{2}^{*}\right] \subset A^{*} \subset K[x, y, z, t]$, since $\left(f_{1}-f_{2}\right)^{*}=(y+z)$ is contained in $A^{*}$, but not in the subalgebra $K\left[f_{1}^{*}, f_{2}^{*}\right]=K\left[x^{3}+y t^{2}, x^{3}-z t^{2}\right]$ which does not contain any homogenous polynomial of degree 1 .

Theorem 3.3. Let $A$ be a subalgebra in $R$ and $B$ be a homogenous subalgebra in $R[t]$.

(a) $\left(A^{*}\right)_{*}=A$.

(b) For a homogenous polynomial $F \in R[t]$. If $F \in A^{*}$ then $F=t^{\alpha} f^{*}$ for $f \in A$ and $\alpha \in \mathbb{N}$.

(c) If $B$ is a homogenous algebra in $R[t]$, then for each $h \in B_{*}$, there is some homogenous element $F \in B$ such that $F_{*}=h$.

Proof. (a) Obviously, we have $A \subset\left(A^{*}\right)_{*}$. Now let $f \in\left(A^{*}\right)_{*}$, by definition the polynomial is of the form $f=\left(\sum_{i=1}^{r} c_{i} m_{i}\left(A^{*}\right)\right)_{*}$ where $m_{i}(A) \in \operatorname{Mon}(A)$ and $c_{i} \in K$. It follows that $f=\sum_{i=1}^{r} c_{i} m_{i}(A)$ which implies that $f \in A$.

(b) If $F \in A^{*}$, we have $F_{*} \in\left(A^{*}\right)_{*}=A$. Given $F \in R[t]$ and $F_{*} \in A$. We obtain $F=t^{\alpha} f^{*}$ for $f=F_{*} \in A$ and $\alpha \in \mathbb{N}$.

(c) We know $B_{*}$ is the homomorphic image of $B$. There exist a polynomial $F \in B$ such that $F_{*}=f$. By multiplying the homogenous component by a monomial in " $t$ ", we may assume that $F$ is a homogenous polynomial in $B$ which satisfies $F_{*}=f$.

The next lemma describes the behavior of the leading monomials under homogenization and dehomogenization.

Lemma 3.4. The following statements hold:

(a) If $f \in R$, then $L M_{>}(f)=L M_{>}(L H(f))$.

(b) If $f \in R$, then $L M_{>_{t}}\left(f^{*}\right)=L M_{>}(f)$.

(c) If $F$ is a nonzero homogenous element of $R[t]$, then $L M_{>}\left(F_{*}\right)=$ $L M_{>_{t}}(F)_{*}$.

For proofs see $[3,7]$.

The next two theorems examine the behavior of Sagbi basis of subalgebras under homogenization and dehomogenization. 
Theorem 3.5. Let $A=K[G]$ be the subalgebra of $R$ generated by a subset $G$ and $A^{*}$, the homogenization of the subalgebra $A$ in $R[t]$ with respect to $t$. The following two statements are equivalent

(i) $G$ is a Sagbi basis for $A$ in $R[t]$ with respect to monomial ordering $>$.

(ii) $G^{*}=\left\{g^{*} \mid g \in G\right\}$ is a Sagbi basis for $A^{*}$ in $R[t]$ with respect to $>_{t}$.

Proof. (i) $\Rightarrow$ (ii) We have to prove that $K\left[L M_{>_{t}}\left(G^{*}\right)\right]=K\left[L M_{>_{t}}\left(A^{*}\right)\right]$ in order to see that $G^{*}$ is a Sagbi basis for $A^{*}$. For this purpose, it suffices to show that for $F \in A^{*}$ there exist $m\left(G^{*}\right) \in \operatorname{Mon}\left(G^{*}\right)$ such that $L M_{>_{t}}(F)=$ $L M_{>_{t}}\left(m\left(G^{*}\right)\right)$. Since $L M_{>_{t}}(F)=L M_{>_{t}}(L H(F)$ ) (Lemma 3.4(a)), we may assume (without loss of generality) that $F$ is a homogenous polynomial. Also, $F$ can be written as $t^{\alpha} \widetilde{F}$ such that $\widetilde{F} \in A^{*}$ and $t^{\alpha}$ does not divide $\widetilde{F}$. Since $F_{*}=\widetilde{F}_{*}$, we may assume that $F$ is not divisible by $t^{\alpha}$ for any $\alpha \in \mathbb{N} \backslash\{0\}$. By Theorem 3.3(a), $F_{*}=\left(A^{*}\right)_{*}=A$ and $G$ is a Sagbi basis of $A$, therefore $L M_{>}\left(F_{*}\right)=L M_{>}(m(G))$ for some $m(G) \in M o n(G)$. By Lemma 3.4(b), $L M_{>}\left(F_{*}\right)=L M_{>_{t}}\left(\left(F_{*}\right)^{*}\right)$ and $L M_{>}(m(G))=L M_{>_{t}}\left(\left(m\left(G^{*}\right)\right)\right.$. Since $\left(F_{*}\right)^{*}=$ $F$, we obtain $L M_{>_{t}}(F)=L M_{>_{t}}\left(m\left(G^{*}\right)\right)$.

(ii) $\Rightarrow$ (i) Suppose $G^{*}$ is a Sagbi basis for the homogenization algebra $A^{*}$ of $A$ in $R[t]$. Let $f \in A$, then $f^{*} \in A^{*}$. Therefore $L M_{>_{t}}\left(f^{*}\right)=L M_{>_{t}}\left(\left(m\left(G^{*}\right)\right)\right.$ for some $m\left(G^{*}\right) \in \operatorname{Mon}\left(G^{*}\right)$. Since $L M_{>}(f)=L M_{>_{t}}\left(f^{*}\right)$ and $L M_{>}(m(G))=$ $L M_{>_{t}}\left(\left(m\left(G^{*}\right)\right)\right.$, it follows that

$$
L M_{>}(f)=L M_{>}(m(G)) \in K\left[L M_{>}(G)\right]
$$

This shows that $K\left[L M_{>}(A)\right]=K\left[L M_{>}(G)\right]$, i.e $G$ is a Sagbi basis for $A$ in $R$.

Theorem 3.6. Let $B$ be a homogenous subalgebra of $R[t]$. If $G$ is a homogenous Sagbi basis of $B$ with respect to $>_{t}$, then $G_{*}=\left\{F_{*} \mid F \in G\right\}$ is a Sagbi basis for the subalgebra $B_{*}$ with respect to $>$.

Proof. If $G$ is a Sagbi basis of $B$, then $G$ generates $B$ and hence $G_{*}=\phi(G)$ generates $B_{*}=\phi(B)$. By Theorem 3.3(c), for a non-zero $f \in B_{*}$ there exist a homogenous element $H \in B$ such that $H_{*}=f$. It follows from Lemma 3.4(b) that

$$
L M_{>}(f)=L M_{>_{t}}\left(f^{*}\right)=L M_{>_{t}}\left(\left(H_{*}\right)^{*}\right)
$$

On the other hand, there exist $m(G) \in \operatorname{Mon}(G)$ such that

$$
L M_{>_{t}}(H)=L M_{>_{t}}(m(G))
$$

We also have $t^{\alpha}\left(H_{*}\right)^{*}=H$ for $\alpha \in \mathbb{N}$ and hence

$$
L M_{>_{t}}(H)=L M_{>_{t}}\left(t^{\alpha}\left(H_{*}\right)^{*}\right)=t^{\alpha} L M_{>_{t}}\left(\left(H_{*}\right)^{*}\right)
$$


So (3.1), (3.2) and (3.3) yields

$$
L M_{>_{t}}(m(G))=L M_{>_{t}}(H)=t^{\alpha} L M_{>_{t}}\left(\left(H_{*}\right)^{*}\right)=t^{\alpha} L M_{>}(f) .
$$

Taking the dehomogenization for the above equality and using Lemma 3.4(c) we obtain

$$
L M_{>}\left(m\left(G_{*}\right)\right)=L M_{>_{t}}(m(G))_{*}=L M_{>}(f)
$$

This shows that, $G_{*}$ is a Sagbi basis for $A_{*}$.

Corollary 3.7. Let $A$ be a subalgebra of $R$. If $G$ is homogenous Sagbi basis of $A^{*}$ in $R[t]$ with respect to $>_{t}$ then $G_{*}$ is Sagbi basis for $A$ in $R$ with respect to $>$.

Proof. Put $B=A^{*}$. Since $B_{*}=A$, it follows from Theorem 3.6 if $G$ is a homogenous Sagbi basis of $B$ then $G_{*}$ is a homogenous Sagbi basis for $A$.

Let $A=K[G]$ be the subalgebra of $R$ generated by a subset $G$, we know that $K\left[G^{*}\right] \subset A^{*}$ (Example 3.2). By computing homogenous Sagbi basis of $K\left[G^{*}\right]$, we can obtain a Sagbi basis for the subalgebra $A=K[G]$ (Theorem 3.6) and consequently a homogeneous Sagbi basis for $A^{*}$ (Theorem 3.5).

The next theorem shows, how s-reduced Sagbi basis behave under homogenization.

Theorem 3.8. Let $A$ be a subalgebra in $R$. If $G$ is the s-reduced Sagbi basis of $A$ with respect to $>$ then $G^{*}$ is the s-reduced Sagbi basis of $A^{*}$ with respect to $>{ }_{t}$.

Proof. As $G$ is a Sagbi basis of $A$, it follows from Theorem 3.5, that $G^{*}$ is a Sagbi basis of $A^{*}$. Now we have to show that $G^{*}$ is s-reduced. For all $g \in G, L C_{>}(g)=1$ therefore $L C_{>_{t}}\left(g^{*}\right)=1$. If there exists $g^{*} \in G^{*}$ such that $L M_{>_{t}}\left(g^{*}\right) \in K\left[L M_{>_{t}}\left(G^{*} \backslash\left\{g^{*}\right\}\right)\right]$ i.e $L M_{>_{t}}\left(g^{*}\right)=m\left(L M_{>_{t}}\left(G^{*} \backslash\left\{g^{*}\right\}\right)\right)$ for some $m \in \operatorname{Mon}(P)$, from here we get $L M_{>}(g)=L M_{>}(m(G \backslash\{g\})$ (Lemma $3.4(\mathrm{~b}))$, which is a contradiction as $G$ is the s-reduced Sagbi basis.

If for some $g^{*} \in G^{*}, \operatorname{support}\left(g^{*}-L M_{>_{t}}\left(g^{*}\right)\right) \cap K\left[L M_{>_{t}}\left(G^{*}\right)\right] \neq \varnothing$, it implies that there exists $v \in \operatorname{support}\left(g^{*}-L M_{>_{t}}\left(g^{*}\right)\right)$ such that $v=m\left(L M_{>_{t}}\left(G^{*}\right)\right)$, for some $m \in \operatorname{Mon}(P)$. Now $v \in \operatorname{support}\left(g^{*}-L M_{>_{t}}\left(g^{*}\right)\right)$ if and only if $v=t^{\alpha} u$ for $u \in \operatorname{support}\left(g_{i}-L M_{>}\left(g_{i}\right)\right)$ and $\alpha \in \mathbb{N}$. Therefore, $t^{\alpha} u=m\left(L M_{>_{t}}\left(G^{*}\right)\right)$. Taking dehomogenizing, we will obtain $u=$ $m\left(L M_{>}(G)\right)$ which is a contradiction as $G$ is the s-reduced Sagbi basis.

The next example shows that for homogenous subalgebra $B \subset R[t]$, the demogenization of the s-reduced Sagbi basis of $B$ is not necessarily the sreduced Sagbi basis of $B_{*}$. 
Example 3.9. Let $B=K[H] \subset K[x, y, t]$ where $H=\{x y t, x y+y t+x t, x+$ $y+t\}$. The set $H$ is the homogenous s-reduced Sagbi basis of the homogenous subalgebra $B$ with respect to the degree lexicographical ordering. The set $H_{*}=$ $\{x y, x y+y+x, x+y+1\}$ is a Sagbi basis of $B_{*}$ with respect to the degree lexicographical ordering but $H_{*}$ is not the s-reduced Sagbi basis of $B_{*}$.

\section{Acknowledgment:}

I wish to thanks Prof. Dr. Gerhard Pfister for his constant support and valuable suggestions.

\section{References}

[1] B. Buchberger, Ein Algorithmus zum Aunden der Basiselemente des Restklassenrings nach einem nulldimensionalen Polynomideal, $\mathrm{PhD}$. Thesis, Inst. University of Innsbruck, Innsbruck, Austria, (1965).

[2] B. Buchberger, Gröbner Bases: An Algorithmic Method in Polynomial Ideal Theory, Multidimensional Systems Theory (N.K. Bose, ed.), Reidel, Dordrecht, (1985), 184-232.

[3] G-M Greuel, G. Pfister, A SINGULAR Introduction to Commutative Algebra, Springer, second edition, (2008).

[4] G-M Greuel, G. Pfister and H. Schönemann, SINGULAR - A Computer Algebra System for Polynomial Computations, Free software under GNU General Public Licence, (1990-to date).

[5] D. Kapur, K. Madlener, A Completion Procedure for Computing a Canonical Basis for a k- Subalgebra. Computers and Mathematics, Springer, New York, (1989), 1-11.

[6] M. Kreuzer, L. Robbiano, Computational Commutative Algebra 2, Springer-Verlag, (2005).

[7] H. Li, Note on (De)homogenize Gröbner Bases. Journal of Algebra, Number Theory: Advances and Applications, 1(3)(2010), 35-70.

[8] J. Lyn Miller, Analogs of Gröbner bases in Polynomial Rings over a Ring, J. Symbolic Computation 21(1996), 139-153.

[9] D. Popesu, Bounds of Stanley depth, An. St. Univ. Ovidius Constanta, Vol 19(2)(2011), 187-194.

[10] L. Robbiano, On the Theory of Graded Structures, J. Symbolic Computation 2(1986), 139-170. 
[11] L. Robbiano, M. Sweedler, Subalgebra Bases, Lectures Note in Mathematics series, Springer-Verlag, Volume 1430(1988), 61-87.

Junaid Alam Khan,

Department of Mathematical Sciences,

Institute of Business Administration,

University Road, Karachi, Pakistan.

Email: junaidalamkhan@gmail.com 